\title{
Temperature Effects on Developmental Stages of Isolates from Three Clonal Lineages of Phytophthora infestans
}

\author{
Eduardo S. G. Mizubuti and William E. Fry
}

Department of Plant Pathology, Cornell University, Ithaca, NY 14853.

Accepted for publication 17 April 1998.

\begin{abstract}
Mizubuti, E. S. G., and Fry, W. E. 1998. Temperature effects on developmental stages of isolates from three clonal lineages of Phytophthora infestans. Phytopathology 88:837-843.

Sporangia germination of Phytophthora infestans isolates belonging to three clonal lineages (US-1, -7, and -8) was assessed at temperatures ranging from 10 to $25^{\circ} \mathrm{C}$. At $10^{\circ} \mathrm{C}$ there were no significant differences in germination percents among US-1, -7 , and -8 . At 18 or $20^{\circ} \mathrm{C}$ US-7 and -8 had significantly lower germination percents than US-1. At 21,24 , or $25^{\circ} \mathrm{C}$ all clonal lineages had low germination percents. Sporangia of the US-7 and -8 lineages germinated more quickly at $15^{\circ} \mathrm{C}(P=0.001)$ dur-

isolates were assessed on inoculated detached leaflets of susceptible potato cv. Norchip kept at $10,15,20$, or $25^{\circ} \mathrm{C}$. In general, IP declined exponentially and LA increased exponentially with increasing temperatures. SPU had a quadratic shape, with the maximum at $15^{\circ} \mathrm{C}$. Averaged over all temperatures, the US-7 lineage had the shortest IP (59.3 $\mathrm{h}$ compared to $66.4 \mathrm{~h}$ for US-1 $[P=0.012]$ and $71.7 \mathrm{~h}$ for US-8 $[P=0.026])$. Again, averaged over all temperatures, the US-8 lineage had a larger LA $(P=$ 0.030 ) than US-1. There was no significant difference between US-7 and -1 for LA. There were no significant differences among lineages in terms of SPU. These results indicate that clonal lineages differ from each other in epidemiological attributes, but the differences can be complex.
\end{abstract} ing the first $2 \mathrm{~h}$ than did the US-1 lineage. The incubation period (IP), lesion area (LA), and sporulation per unit of lesion area (SPU) of the

Late blight of potato, caused by Phytophthora infestans (Mont.) de Bary, has been an important disease since the mid-nineteenth century, when it caused the Irish Potato Famine, and considerable crop losses still occur. Recently, late blight has reemerged as an important disease throughout the world (9).

Late blight epidemics are associated with wet conditions and moderate temperatures $(20,23)$. Important events in the pathogen's life cycle are influenced by major weather variables, such as moisture and temperature $(8,19)$. Moisture (as free moisture or relative humidity) is important during sporulation, zoosporogenesis, sporangia and zoospore germination, and sporangia survival $(11,21,23$, $33,35,43)$. Temperature can noticeably influence spore germination $(5,31,38)$, mycelial growth rate $(22)$, inoculum production $(37$, $44)$, and survival $(7,36,37)$.

The effect of temperature on sporangia germination has been observed since the pioneering work of De Bary during the 19th century (6), but currently, most of the information on how temperature affects sporangia germination is derived from studies done by Melhus (31) and Crosier (5) during the early 20th century. Temperatures lower than $20^{\circ} \mathrm{C}$, with the optimum between 12 to $13^{\circ} \mathrm{C}$, favor indirect germination of sporangia, i.e., formation of zoospores (5 to 10 per sporangium). Temperatures higher than $20^{\circ} \mathrm{C}$, with the optimum at $24^{\circ} \mathrm{C}$, favor direct germination, i.e., one sporangium gives rise to a germ tube. Even under optimum temperatures, the percentage of direct germination is usually much lower than the percentage of indirect germination $(5,31)$. Although not explicitly stated, it appears that all experiments done by Melhus (31) and Crosier (5) were based on just one isolate of $P$. infestans.

Until recently, the $P$. infestans population around the world apparently was composed of a single clonal lineage of the A1 mating

Corresponding author: W. E. Fry; E-mail address: wef1 @ cornell.edu

Publication no. P-1998-0526-01R

(C) 1998 The American Phytopathological Society
Additional keywords: environment, late blight.

type (10). If a single clonal lineage was present in Europe, Canada, and the United States (13), then all information about temperature effects on pathogen development was gathered using this single lineage of $P$. infestans. This scenario has changed. "New" clonal lineages (sensu Goodwin et al. [13]) are taking over and displacing the "old" clonal lineage in several locations worldwide (12). It is unknown whether the new clonal lineages differ in temperature requirements from those previously described for the old clonal lineage.

The concept of clonal lineage has had great utility for understanding the population biology of $P$. infestans in most locations in the United States and Canada during the 1990s. The isolates within lineages are more similar to each other than to other isolates for characteristics such as metalaxyl resistance, specific virulence, aggressiveness, and host preference $(16,17,28,30,32)$. The major mechanism for diversity within a clonal lineage is mutation, and older lineages are expected to be more diverse than younger lineages. This expectation was tested and found to occur with specific virulence in $P$. infestans $(16,41)$. Thus, knowledge of clonal lineage provides some predictive value about a particular isolate for some ecologically important attributes. We do not know whether knowledge of clonal lineage also might provide information concerning effects of temperature on developmental stages.

Small variations in optimum ranges of weather variables can affect epidemic development. For instance, epidemics developing at slightly lower mean temperatures during the night (e.g., 12 to $16^{\circ} \mathrm{C}$ ) are expected to be more severe because of the effect this temperature range has on sporangia germination. Indirect germination of sporangia is favored under this temperature range, increasing infective potential and, consequently, epidemic development.

It is possible that genotypes of $P$. infestans may be influenced differentially by a change in temperature. Thus, to ascertain whether a particular isolate or a clonal lineage as a whole has unique temperature requirements for sporangia germination or whether ag- 
gressiveness is temperature dependent, it is important to analyze these two attributes under different temperatures.

The objective of the current study was to determine whether different clonal lineages of $P$. infestans respond similarly to temperature. Our hypothesis was that all clonal lineages would respond similarly to temperature. The approach taken to test this hypothesis was to measure sporangia germination, incubation period (IP), lesion area (LA), and sporulation per unit of lesion area (SPU) for several isolates from each of three clonal lineages (US-1, -7, and8 ) at different temperatures. If significant differences were detected, adjustments in disease management might be needed.

\section{MATERIALS AND METHODS}

Clonal lineages and isolates. The US-1 clonal lineage is the old $P$. infestans clonal lineage, and clonal lineages US-7 and -8 are two of the new clonal lineages of $P$. infestans (13). For all experiments, isolates from the clonal lineages were obtained from the $P$. infestans culture collection at Cornell University, Ithaca, NY. To reduce uncontrolled variance, all isolates were collected in 1994, primarily from the northeastern United States. During July 1995, the isolates were taken out of storage in a deep freezer $\left(-130^{\circ} \mathrm{C}\right)$ and transferred to rye B agar medium (2). To confirm the genotype of each isolate, DNA fingerprints, using RG57 probe (14), and isozyme profiles (15) were determined. In each case, the preliminary identification of lineage was confirmed.

Inoculum production. Potato plants of highly susceptible cv. Norchip were grown in a greenhouse (night temperature $18^{\circ} \mathrm{C}$; day temperature $25^{\circ} \mathrm{C}$ ) with the photoperiod extended to $14 \mathrm{~h}$, using $400 \mathrm{~W}$ GE Lucalox lamps (General Electric Co., Schenectady, NY). Leaflets were obtained from 4- to 8-week-old plants and placed in inverted $15-\mathrm{cm}$ petri plates. A layer of water agar in the bottom portion served as the moisture source above each leaflet (30). There were three leaflets per plate. Sporangia from 10- to 14-day-old cultures of $P$. infestans grown on rye B agar medium (2) at $18^{\circ} \mathrm{C}$ were washed with a salt solution (39). The sporangia were collected, and the suspension concentration was adjusted to $20,000 / \mathrm{ml}$ with a hemacytometer. Sporangial suspensions were prepared as rapidly as possible, so sporangia were not in the salt solution for more than $120 \mathrm{~min}$ before inoculation. Each of the three leaflets in a single petri dish was inoculated with a $50-\mu \mathrm{l}$ drop of a sporangial suspension of a single isolate. Thus, each leaflet received $\approx 1,000$ sporangia. Inoculated leaves in moist chambers were placed in a growth chamber under weak light $(0.02$ to $0.03 \mu \mathrm{E} \mathrm{m}^{-2} \mathrm{~s}^{-1}$ ) at $18^{\circ} \mathrm{C}$ as described previously (26,30). All

TABLE 1. Isolates from the US-1, -7 , and -8 clonal lineages of Phytophthora infestans used in the experiments

\begin{tabular}{|c|c|c|c|c|c|}
\hline Isolate $^{\mathrm{a}}$ & $\begin{array}{l}\text { Clonal } \\
\text { lineage }\end{array}$ & Exp. $1^{\mathrm{b}}$ & Exp. 2 & Exp. 3 & $\begin{array}{c}\text { Fitness } \\
\text { components } \\
\text { experiment }\end{array}$ \\
\hline US940498 & & & + & & + \\
\hline US940499 & US-1 & + & + & & \\
\hline US940501 & US-1 & + & + & + & + \\
\hline US940507 & US-1 & & + & & + \\
\hline US940330 & US-7 & & + & & + \\
\hline US940475 & US-7 & & + & & + \\
\hline US940486 & US-7 & & + & & + \\
\hline US940488 & US-7 & & + & + & + \\
\hline US940477 & US-8 & & + & & + \\
\hline US940480 & US-8 & + & + & + & + \\
\hline US940482 & US-8 & + & & & \\
\hline US940491 & US-8 & & + & & + \\
\hline US940497 & US-8 & & + & & + \\
\hline
\end{tabular}

${ }^{a}$ Reference number from the Cornell University P. infestans culture collection.

${ }^{b}$ Plus indicates the isolate was used $\mathrm{n}$ the experiment. inoculum was produced at $18^{\circ} \mathrm{C}$, a favorable temperature for maturation of sporangia of $P$. infestans (37).

To assess the effect of temperature on sporangia germination, three sets of experiments were conducted. In the first experiment, comparisons were made between isolates from two clonal lineages of $P$. infestans, US- 1 and -8 , at $15,18,21$, and $24^{\circ} \mathrm{C}$. The second experiment was carried out with three clonal lineages, US-1, -7, and -8 , and under a wider temperature range, 10, 15, 20, and $25^{\circ} \mathrm{C}$. The third experiment was designed to investigate the effect of temperature on the speed of sporangia germination at $15^{\circ} \mathrm{C}$. No distinction was made between direct and indirect germination in any of the sporangia germination experiments. However, there was a low percentage of direct sporangia germination.

Because maximum germination differed among lineages, all germination data are presented relative to the maximum germination of the particular lineage in the specific experiment. All maxima occurred in the range of 10 to $15^{\circ} \mathrm{C}$. In general, the maximum for US- 1 occurred at $15^{\circ} \mathrm{C}$, and the maxima for US- 7 and -8 occurred at $10^{\circ} \mathrm{C}$. Maximum germination (percent) was determined for a particular lineage, and relative germination (percentage of maximum) was determined for each lineage-temperature combination. All germination data presented in figures are given as percentages of maxima.

Experiment 1: temperature effect on total sporangia germination of isolates from the US-1 and -8 clonal lineages. Sporangia germination of two isolates (Table 1) from each clonal lineage was assessed at different temperatures. Sporangia from infected leaflets were transferred to water agar $(1.5 \%)$ in petri plates via membrane filters. Transfer was accomplished by gently touching the lesions with a membrane filter (Whatman 47-mm-diameter, $0.45-\mu \mathrm{m}$ pore size, mixed cellulose esters; Whatman International Ltd., Maidstone, England), laying the membrane on the agar surface for $\approx 10 \mathrm{~s}$, and removing the membrane. Plates were placed in incubators at $15,18,21$, or $24^{\circ} \mathrm{C}$ with no light. After $12 \mathrm{~h}$, germination was assessed by observing microscopically $(100 \times)$ a piece of water agar $(2 \times 2 \mathrm{~cm})$ containing sporangia. There were three replicates for each combination of isolate and temperature, and each replicate consisted of an independent petri plate. At least 300 sporangia were counted per replicate.

The experiment was set in a completely randomized design. Total germination refers to sporangia germination, direct and indirect, evaluated after $12 \mathrm{~h}$ of incubation. The experiment was done twice. To satisfy the assumptions of analysis of variance (ANOVA), sporangia germination (percentage of maximum germination) was square-root transformed.

Experiment 2: temperature effect on total sporangia germination of isolates from the US-1, $\mathbf{- 7}$, and $\mathbf{- 8}$ clonal lineages. Sporangia germination of four isolates (Table 1) from each of three clonal lineages of $P$. infestans, US-1, -7, and -8, was assessed after incubation under different temperatures. Sporangia production and transfer proceeded as described for the previous experiment. Petri plates containing water agar and sporangia were placed in incubators at $10,15,20$, or $25^{\circ} \mathrm{C}$ with no light. After $12 \mathrm{~h}$, germination was assessed by microscopic observation (100X) of a piece of water agar containing sporangia. There were three replicates for each combination of isolate and temperature, and each replicate consisted of an independent petri plate. At least 300 sporangia were counted per replicate. The experiment was set in a randomized complete block design. Each block was an independent day on which the experiment was done. Total germination refers to sporangia germination, direct and indirect, evaluated after $12 \mathrm{~h}$ of incubation.

Experiment 3: speed of sporangia germination at optimum temperature. To determine whether there were differences in speed of germination, sporangia were observed every $2 \mathrm{~h}$ at $15^{\circ} \mathrm{C}$. This temperature was chosen as the common favorable temperature for sporangia germination of all isolates from the three clonal lineages (discussed below). One isolate from each clonal lineage was cho- 
sen. Sporangia were observed at 2, 4, 6, and $8 \mathrm{~h}$ after incubation. A completely randomized design was used with three replicates, each replicate consisting of one petri plate. At least 300 sporangia were counted per replicate. The experiment was done twice.

Temperature effect on fitness components. The effect of temperature on fitness components was assessed for isolates from the US-1 (three isolates), US-7 (four isolates), and US-8 (four isolates) clonal lineages on detached leaflets of potato. Detached leaflet assays used here reflect isolate differences observed in field experiments $(29,30)$. Additionally, detached leaflet studies have identified differences among potato genotypes in breeding programs (18). The 11 isolates selected for this experiment are listed in Table 1.

Inoculation. Inoculum consisted of sporangia obtained from 6-day-old sporulating lesions from inoculated leaflets as described above. Sporangia were collected in small beakers with $10 \mathrm{ml}$ of a salt solution (39). Sporangia concentration was adjusted to $5 \times 10^{3}$ sporangia per $\mathrm{ml}$. At least two hemacytometer readings were used when determining sporangia concentration. Before inoculation, the sporangia suspensions were kept at $4^{\circ} \mathrm{C}$ for $40 \mathrm{~min}$ to enhance zoospore release.

Two leaflets, abaxial side up, were placed in each small moist chamber. Each leaflet was inoculated with one 50- $\mu$ d droplet of sporangia suspension. One small moist chamber with two leaflets was used for each isolate-temperature combination.

Incubation conditions. After inoculation, each plate was sealed with three layers of Parafilm to prevent desiccation during incubation. Moist chambers containing inoculated leaves were distributed randomly inside growth chambers set at four temperatures: 10, 15, 20 and $25^{\circ} \mathrm{C}$ with a 14 -h photoperiod. A randomized complete block design with three blocks was used. Each block was a different inoculation date.

Assessments. For IP, assessments were taken at 36, 42, 48, 54, $60,66,72,78,84,90,96,108,114$, and $120 \mathrm{~h}$ (5 days) after inoculation (HAI) by visually inspecting the lesions. The IP of an individual lesion was considered completed when small necrotic spots were observed at the inoculation site. To satisfy the assumptions of ANOVA, the variable IP was logarithmically transformed.

The LA for each isolate-temperature combination was assessed with the aid of a video camera. All lesions were recorded at 140 HAI ( $\approx 6$ days). Images were captured, digitized, and saved as computer files for future analysis. LA measurements were accomplished with ImageTool software (UTHSCSA, University of Texas Health Science Center, San Antonio). The variable LA was logarithmically transformed for ANOVA.

Sporulation was assessed at $140 \mathrm{HAI}$. Lesions were cut from leaflets and transferred to screw-cap plastic vials containing $5 \mathrm{ml}$ of a preservation solution $(2.5 \mathrm{ml}$ of distilled water and $2.5 \mathrm{ml}$ of $0.04 \mathrm{M} \mathrm{CuSO}_{4} / 0.2 \mathrm{M}$ sodium acetate/acetic acid, $\mathrm{pH}$ 5.4). Sporangia were washed from lesions by vortexing the vials for $10 \mathrm{~s}$. Plant tissue was removed, and vials were stored at $4{ }^{\circ} \mathrm{C}$. Sporangia were counted within $48 \mathrm{~h}$ with a hemacytometer. At least two hemacytometer readings were done for each sporangial suspension. Sporangia production per unit of lesion area was calculated by multiplying the sporangia concentration per milliliter (determined with the hemacytometer) by the volume of preservation solution and dividing by total lesion area (square centimeters). The variable SPU was square-root transformed.

Statistical analysis. ANOVA was used for all data with different linear models. For temperature effect on sporangia germination (experiment 2) and fitness components, the treatment design was a nested-crossed factorial treatment design. The linear model was

$$
Y_{i j k l m}=\mu+\alpha_{i}+\beta_{j}+\alpha \beta_{i j}+\gamma_{k(i)}+\beta \gamma_{j k(i)}+\delta_{l}+e_{i j k l m}
$$

in which $\mu$ is the overall mean, $\alpha_{i}$ is the fixed effect of clonal lineage, $\beta_{j}$ is the fixed effect of temperature, $\alpha \beta_{i j}$ is the interaction term between clonal lineage and temperature, $\gamma_{k(i)}$ is the fixed effect of isolates nested within clonal lineages, $\beta \gamma_{j k(i)}$ is the interaction term between temperature and isolates within clonal lineages, $\delta_{l}$ is the effect of blocks, and $e_{i j k l m}$ is the random experimental error. This model allowed us to test for differences among clonal lineages, as well as among isolates within clonal lineages. For analysis of experiment 1, a similar model for completely randomized designs was used (27,34). Experiment 3 was analyzed as a completely randomized design in a factorial (clonal lineages $\times$ time) treatment design.

All preplanned comparisons of treatment means, for instance US-1 versus US-8 or old versus new clonal lineages, regarding a particular variable of interest (germination, latent period, lesion area, etc.) were tested, using contrasts (40). All statistical analyses were done with Statistical Analysis Systems, version 6.11 (SAS Institute Inc., Cary, NC).

\section{RESULTS}

Experiment 1: temperature effect on total sporangia germination of isolates from the US-1 and $\mathbf{- 8}$ clonal lineages. Germination of sporangia of isolates from the US-1 and -8 clonal lineages did not respond similarly to temperature. There was a significant differential effect of temperature on sporangia germination for the two clonal lineages, as reflected by a highly significant interaction term between temperature and clonal lineages $(P=0.001)$. A sharper decrease in total sporangia relative germination percents for US-8 compared to US-1 was observed at temperatures ranging from 15 to $24^{\circ} \mathrm{C}$. The mean germination percents for US- 1 and -8 were compared, using contrasts, at each temperature. At $18^{\circ} \mathrm{C}$ there was a drastic reduction in the relative germination percent for the isolates from the US-8 clonal lineage in relation to US-1. The US- 8 clonal lineage had significantly $(P<0.001)$ lower $(8.3 \%)$ germination percents than US-1 isolates $(41.7 \%)$. At 21 and $24^{\circ} \mathrm{C}$ the relative germination percent for isolates belonging to both lineages was low but not significantly different $(P=0.705$ and 0.277 , respectively).

Experiment 2: temperature effect on total sporangia germination of isolates from the US-1, $\mathbf{- 7}$, and $\mathbf{- 8}$ clonal lineages. There was a significant differential effect of temperature on sporangia germination for the three clonal lineages $(P=0.001)$ (Fig. 1). There was no significant difference between the old and new clonal lineages at $10^{\circ} \mathrm{C}(P=0.388)$; however, at 15,20 , and $25^{\circ} \mathrm{C}$ there were significant differences among lineages. At $15^{\circ} \mathrm{C}$, the average relative germination percent for US-1 isolates was significantly higher compared to US-7 and -8 isolates $(P=0.027)$. At $20^{\circ} \mathrm{C}$ germination levels for the isolates from the new clonal lineages, US-7 (5.4\%) and US-8 (8.2\%), were significantly lower compared to isolates from the old clonal lineage, US-1 (62.0\%). The difference in germination percents between the new (US-7 and -8) and old (US-1) clonal lineages was highly significant at $20^{\circ} \mathrm{C}(P=0.001)$. At $25^{\circ} \mathrm{C}$ all clonal lineages had low germination levels, but US-1 had a significantly $(P=0.006)$ higher percent germination than the US-7 and -8 clonal lineages. There were no significant differences among isolates within clonal lineages $(P=0.267)$. In general, germination percents were high at lower temperatures and declined with increasing temperatures (Fig. 1).

Experiment 3: speed of sporangia germination. There was a significant interaction $(P=0.001)$ between time and germination levels for the three isolates representing the three clonal lineages, indicating that germination levels at a given time vary for the three isolates (Fig. 2). At $2 \mathrm{~h}$ of incubation, the average relative germination percents for US-1, -7 , and -8 isolates were $46.3,75.8$, and $80.9 \%$, respectively. The germination percent for the old clonal lineage (US-1) isolate was significantly lower $(P=0.001)$ than that of the new clonal lineage (US-7 and -8) isolates. The difference between germination level for isolates from the old and new 
clonal lineages at $4 \mathrm{~h}$ of incubation also was significant $(P=0.043)$, mainly due to a significantly higher germination level for the US-7 isolate in relation to the US-1 isolate $(P=0.021)$. The difference at 6 and $8 \mathrm{~h}$ of incubation was not significant $(P=0.093$ and 0.074, respectively) (Fig. 2).

Temperature effects on fitness components. There was no significant interaction between clonal lineages and temperature for IP, LA, or SPU ( $P=0.067,0.526$, and 0.139 , respectively). The lack of significance of the interaction term allowed us to investigate the main effects of clonal lineages and temperature independently $(27,40)$. The means and standard errors of all measured variables, IP, LA, and SPU, of each clonal lineage at each of the temperatures are shown in Table 2. The IP was shortest for US-7 isolates, with a mean of $59.3 \mathrm{~h}$ compared to $66.4 \mathrm{~h}$ for US-1 and $71.7 \mathrm{~h}$ for US-8. The IP for the US-7 clonal lineage was significantly shorter than that of US-1 $(P=0.012)$, and the IP for US-8 was significantly $(P=0.026)$ longer than that of US-1. Averaged over all temperatures, isolates from the US- 8 lineage had a significantly larger LA than US-1 $(P=0.030)$. There was no significant difference between US-7 and -1 for LA $(P=0.506)$. There was no significant difference between either US-1 and $-7(P=0.492)$ or US-1 and $-8(P=0.637)$ for SPU. There were no significant differences among isolates within clonal lineages for IP, LA, or SPU $(P=0.242,0.118,0.198$, respectively).

In general, IP declined approximately exponentially (Fig. 3A) and LA increased approximately exponentially (Fig. 3B) with increasing temperatures. SPU had a quadratic shape, with the maximum at $15^{\circ} \mathrm{C}$ (Fig. 3C). The effect of temperature was significant $(\alpha=$ $0.01)$ for all measured fitness components. The models relating fitness components as a function of temperature (Fig. 3) were significant $(\alpha=0.05)$.

\section{DISCUSSION}

Temperature had a striking differential effect on sporangia germination rates for the different lineages of $P$. infestans. Isolates from the US-1 clonal lineage, in general, had a higher final germination percent (after $12 \mathrm{~h}$ of incubation) for all temperatures higher than $10^{\circ} \mathrm{C}$; the difference at $20^{\circ} \mathrm{C}$ was the largest, but differences also were evident at $18^{\circ} \mathrm{C}$. Temperatures higher than

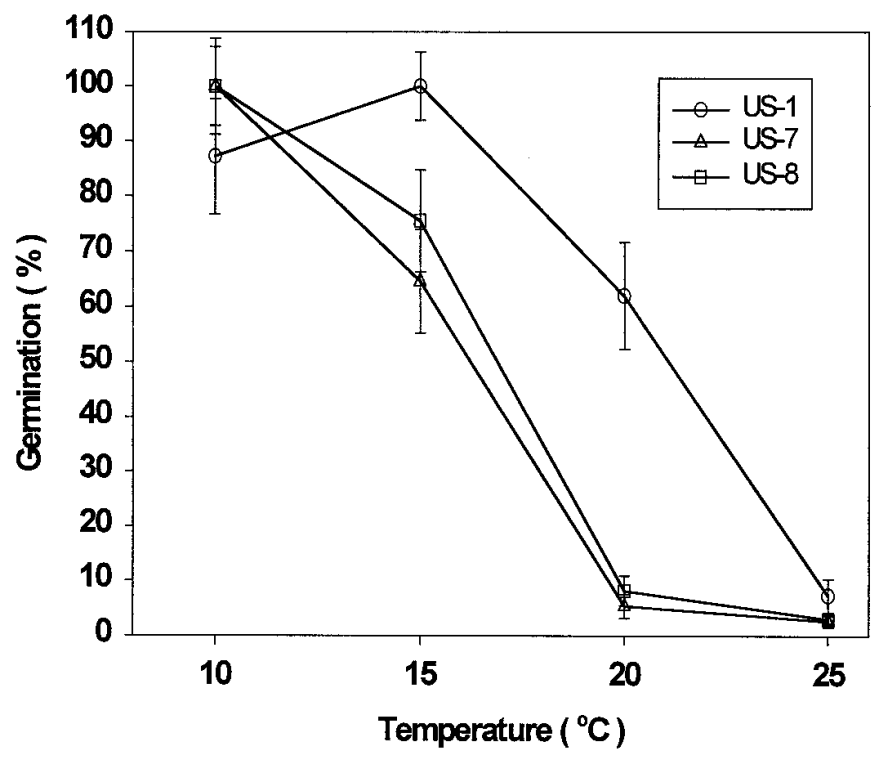

Fig. 1. Relative sporangia germination percents for US-1, -7, and -8 clonal lineages of Phytophthora infestans under different temperatures. Maximum absolute average values were $76.5,68.3$, and $67.9 \%$ for US-1, -7 , and -8 , respectively. Each data point represents the average of four isolates from each clonal lineage replicated three times. Vertical bars indicate the standard error of the mean. $18^{\circ} \mathrm{C}$ seem to be unfavorable for sporangia germination of isolates from the new clonal lineages.

Interestingly, our results for temperature effects on germination of sporangia for isolates from the US- 1 clonal lineage very closely resemble the results obtained by Melhus (31), Crosier (5), and Sato (37). In each of these older studies, germination at $20^{\circ} \mathrm{C}$ was reduced to approximately half the maximum germination, which we also found for US-1 isolates (Fig. 1). This result is consistent with the hypothesis that in the previous studies the isolates belonged to the US-1 clonal lineage of $P$. infestans. Clearly, our data for isolates from the US-7 and -8 clonal lineages indicate that these isolates respond differently to temperature in terms of sporangia germination.

The differential effect of temperature on sporangia germination could have important implications for epidemic development and disease management. Sporangia of isolates from the US-7 and -8 lineages germinated better at lower temperatures $\left(\leq 15^{\circ} \mathrm{C}\right)$. This may contribute to higher disease intensity because of indirect germination of sporangia instead of direct germination. Indirect germination boosts infection efficiency because of the larger numbers of infective propagules (8) - each sporangium can produce 5 to 10 zoospores. However, this does not imply advantages for isolates from the new clonal lineages in ability to establish infection, because sporangia of US-1 isolates also have high germination levels at low temperatures.

A faster germination speed can enable an organism to overcome some limitations imposed by the environment. If sporangia from the three clonal lineages used in our experiments land on a potato leaflet, germination at $15^{\circ} \mathrm{C}$ would be faster for sporangia of the new clonal lineages. This characteristic can play an important role in disease development when marginal or short-term favorable weather conditions for sporangia germination occur. Infection and colonization might be more likely for isolates from the new clonal lineages under marginal environmental conditions.

Simulation and forecast systems constructed from information available for the old clonal lineage may need to be adjusted to accommodate the different characteristics of the new lineages of $P$. infestans. It would even be possible to devise lineage-oriented simulation and forecast systems, but this approach might be limited if the clonal structure of the $P$. infestans population changes.

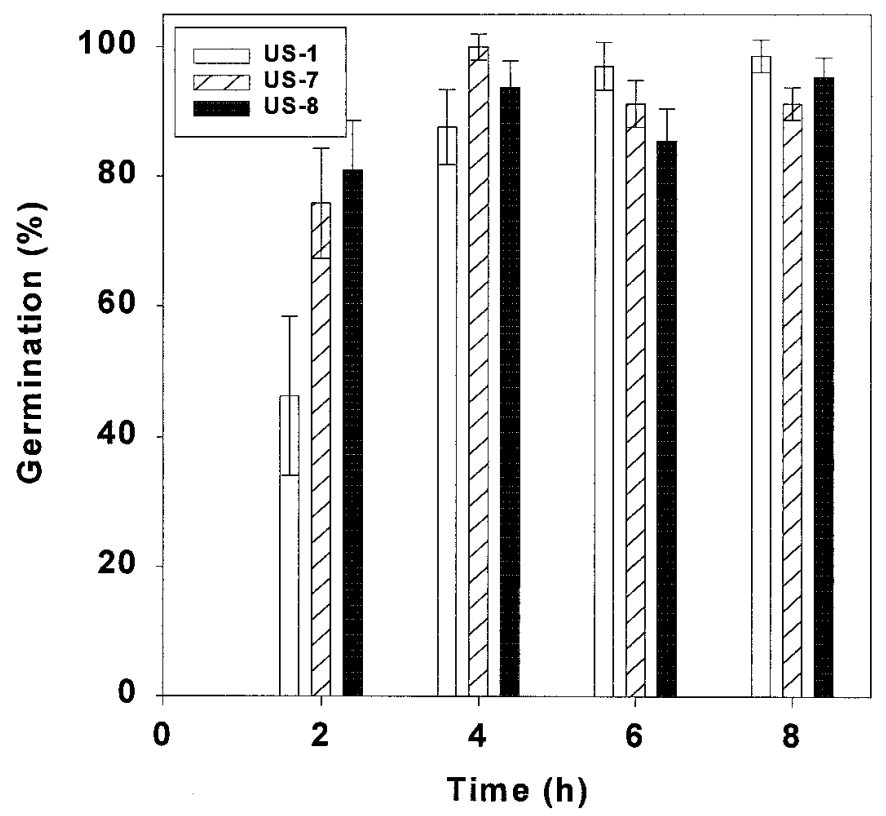

Fig. 2. Relative sporangia germination percents for US-1, -7 , and -8 clonal lineages of Phytophthora infestans at different times after incubation at $15^{\circ} \mathrm{C}$. Maximum absolute average values were $80.1,81.8$, and $80.3 \%$ for US-1, -7 , and -8 , respectively. Vertical bars indicate the standard error of the mean. 
Because sporangia germination in different lineages varies with temperature, caution should be exerted when planning experiments with these clonal lineages of $P$. infestans. If inoculation/incubation is carried out at $20^{\circ} \mathrm{C}$ and sporangia constitute the inoculum, germination rates are expected to be low for the US-7 and -8 clonal lineages. The results could confound treatment effects.

Although temperature had a differential effect on sporangia germination of the isolates from the different clonal lineages of $P$. infestans, its effect on the measured fitness components was not differential. There was no interaction between clonal lineages and temperature for the measured fitness components.

In this study, isolates from the US-7 clonal lineage had the shortest IP among all lineages. A short IP leads to a shorter latent period (LP; the period between inoculation and sporulation). This characteristic enables more reproductive cycles in a growing season, and other aspects being similar, this leads to faster epidemic buildup. Our results are similar to those of an earlier study in which the US-7 clonal lineage also had the shortest LP at $18^{\circ} \mathrm{C}$ (26). Although the experiments cannot be compared directly, because different variables were measured (IP in this paper and LP in the other study), both studies indicate that lesions will appear faster if produced by isolates from the US-7 clonal lineage than if produced by isolates from the US- 1 or -8 clonal lineages.

LA is an important fitness component because it determines the amount of affected leaf tissue (disease severity). If a clonal lineage produces larger lesions, disease intensity is expected to be higher. Remarkably, there was no significant interaction between isolates from the different clonal lineages and temperature, suggesting isolates from the US- 8 clonal lineage have the ability to cause more severe epidemics under a broad range of temperatures. Despite having the longest IP, the final LA for US-8 isolates used in this study was the largest, indicating lesion expansion rate for US-8 isolates is faster than for the other lineages. Kato et al. (26) also found a larger LA for US-8 isolates when LA was assessed at $18^{\circ} \mathrm{C}$, and Chycoski and Punja (3) detected faster growth rates of US-8 isolates compared to US-1 isolates.

SPU was not significantly different among isolates from the different lineages. This result seems to be generally applicable $(3,26)$. Other authors also reported no differences in SPU when comparing metalaxyl-resistant and -sensitive isolates $(24,25)$. Differences in sporulation seem more difficult to detect than differences in other traits, such as IP and LA. This could be due to greater

TABLE 2. Fitness components of three clonal lineages of Phytophthora infestans at different temperatures

\begin{tabular}{|c|c|c|c|c|}
\hline \multirow[b]{2}{*}{ Component $^{\mathrm{a}}$} & \multirow{2}{*}{$\begin{array}{c}\text { Temp. } \\
\left({ }^{\circ} \mathrm{C}\right)\end{array}$} & \multicolumn{3}{|c|}{ Value $\mathrm{b}^{\mathrm{b}}$ clonal lineage } \\
\hline & & US-1 & US-7 & US-8 \\
\hline \multirow[t]{4}{*}{ IP } & 10 & $98.7 \pm 3.6$ & $86.9 \pm 4.2$ & $109.0 \pm 2.5$ \\
\hline & 15 & $59.3 \pm 1.9$ & $56.3 \pm 2.1$ & $65.0 \pm 2.9$ \\
\hline & 20 & $55.7 \pm 2.8$ & $51.3 \pm 2.4$ & $59.5 \pm 3.0$ \\
\hline & 25 & $52.0 \pm 2.8$ & $45.7 \pm 2.1$ & $55.5 \pm 2.6$ \\
\hline Mean $^{\mathrm{c}}$ & & $66.4 \pm 2.6$ & $59.3 \pm 2.1$ & $71.7 \pm 2.6$ \\
\hline \multirow[t]{4}{*}{ LA } & 10 & $0.57 \pm 0.11$ & $0.51 \pm 0.05$ & $0.75 \pm 0.12$ \\
\hline & 15 & $2.63 \pm 0.31$ & $2.35 \pm 0.25$ & $3.32 \pm 0.44$ \\
\hline & 20 & $7.19 \pm 0.83$ & $5.49 \pm 0.51$ & $8.32 \pm 0.76$ \\
\hline & 25 & $9.12 \pm 1.02$ & $7.98 \pm 0.89$ & $12.37 \pm 0.85$ \\
\hline Mean $^{c}$ & & $4.87 \pm 0.52$ & $4.16 \pm 0.40$ & $6.25 \pm 0.55$ \\
\hline \multirow[t]{4}{*}{ SPU } & 10 & $11,316 \pm 4,047$ & $13,434 \pm 2,771$ & $19,916 \pm 3,249$ \\
\hline & 15 & $60,688 \pm 8,614$ & $48,484 \pm 6,624$ & $53,398 \pm 8,475$ \\
\hline & 20 & $37,256 \pm 12,942$ & $32,680 \pm 4,877$ & $36,257 \pm 8,226$ \\
\hline & 25 & $16,309 \pm 6,749$ & $11,458 \pm 7,324$ & $8,914 \pm 1,049$ \\
\hline Mean $^{c}$ & & $31,393 \pm 4,852$ & $27,104 \pm 3,260$ & $29,724 \pm 3,510$ \\
\hline
\end{tabular}

a IP = Incubation period (hours); LA = lesion area (square centimeters); SPU = sporulation per unit of lesion area (sporangia per square centimeter).

${ }^{\mathrm{b}}$ Mean ( \pm standard error).

${ }^{\mathrm{c}}$ Means and standard errors of the means were calculated from original data sets, not from the means at each temperature. Overall means may differ slightly from the average of individual temperature values in the table. variability associated with this component. If different isolates have similar sporulation capacities, then LA will be a critical epidemiological component, as has been reported elsewhere $(1,4,42)$.

The differences among clonal lineages of $P$. infestans are likely to be greater than differences among isolates within lineages. The nonsignificance of the effects of different isolates within clonal lineages for IP, LA, and SPU indicates that isolates belonging to
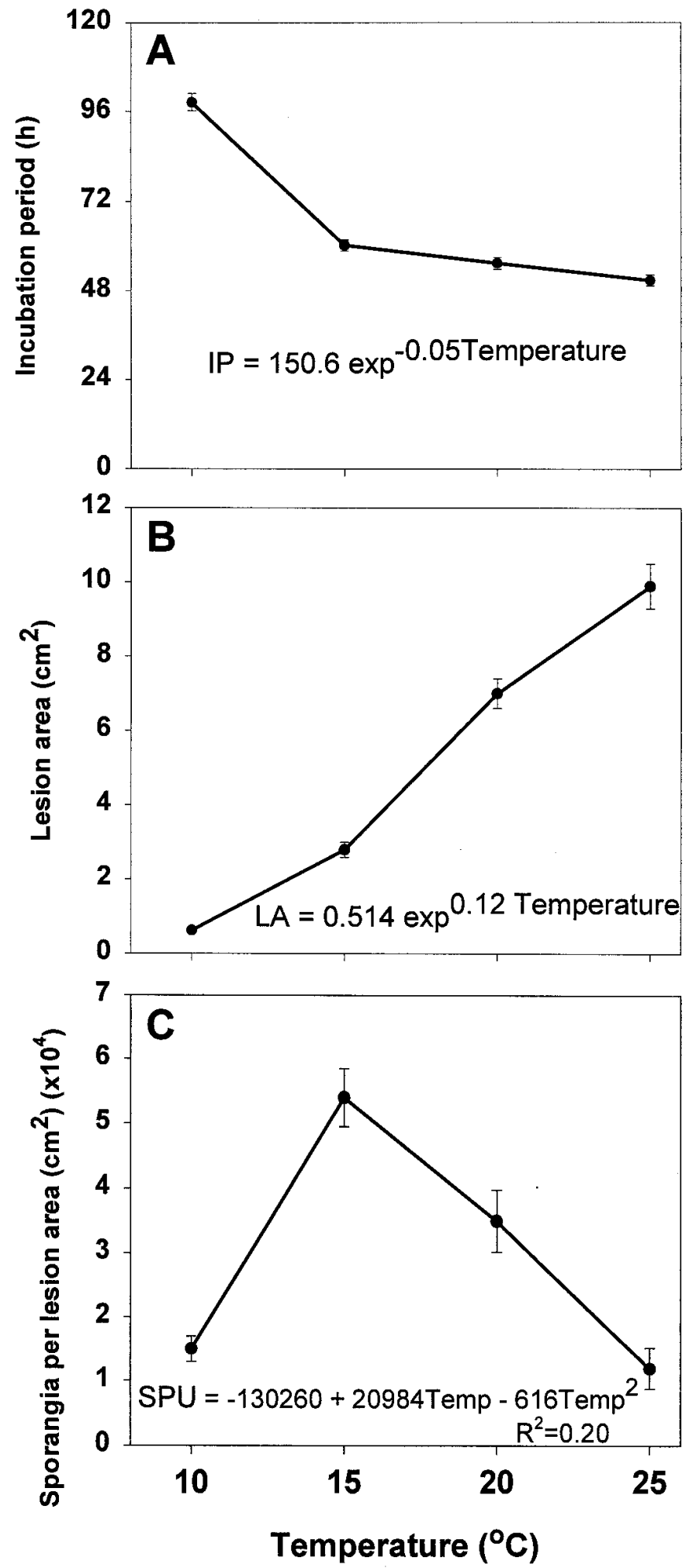

Fig. 3. Effect of temperature on $\mathbf{A}$, incubation period; $\mathbf{B}$, lesion area; and $\mathbf{C}$, sporulation per unit of lesion area for isolates from three Phytophthora infestans clonal lineages. Each data point represents the average of all 11 isolates at each temperature. Vertical bars indicate the standard error of the mean. 
one clonal lineage are more similar to each other than to isolates from different lineages; there is less variability within a clonal lineage than among lineages.

There were two unexpected results found in this study. The first was the narrow favorable temperature range for sporangia germination of isolates from the US-7 and -8 clonal lineages, and the second was a longer IP for the isolates belonging to the US-8 clonal lineage. Initially, these characteristics seem inconsistent with the more severe epidemics caused by the new clonal lineages (9). During the crop season in the northeastern United States, temperatures higher than $20^{\circ} \mathrm{C}$ are common, but temperatures during the night or early morning (when germination is more likely) are frequently lower than $20^{\circ} \mathrm{C}$. Perhaps the more rapid germination rates of the new clonal lineages compensates for the lower optimum temperature range for sporangia germination. Rapid germination would enable infections under marginal conditions. Once infections are established, the faster lesion expansion rates for US-8 compared to US-1 would lead to larger lesions.

Although the detached leaflet approach correlated well with whole plants in the field $(29,30)$, the most accurate inferences about the aggressiveness of the clonal lineages are available only from field experiments. To date, direct comparison is not possible in our experimental area, because both mating types of $P$. infestans are not yet present. Direct comparison of isolates of opposite mating type in the field would provide the opportunity for sexual reproduction. The establishment of a sexually recombining population would be problematic, not only for future work in our experimental area but, potentially, also for potato and tomato growers in the surrounding area.

This study provides data with implications for two aspects of the epidemiology of potato late blight. First, it clearly demonstrates differences among clonal lineages in terms of response to temperature. Differences in temperature effects need to be considered when using simulation or forecast models that were parameterized with data collected based on isolates from the old clonal lineage. Any extrapolation from these models to epidemics caused by isolates from new clonal lineage should be done cautiously. Secondly, this study provides additional experimental data supporting the observations $(3,26,28,32)$ that the new clonal lineages (US-8 in particular) are more aggressive pathogens and, therefore, can cause more severe epidemics.

\section{ACKNOWLEDGMENTS}

Supported in part by NE-IPM grant 96-34103-3333. E. S. G. Mizubuti was supported by CAPES-Brazil. We thank J. Erlich for assistance with germination experiments.

\section{LITERATURE CITED}

1. Birhman, R. K., and Singh, B. P. 1995. Path-coefficient analyses and genetic parameters of the components of field resistance of potatoes to late blight. Ann. Appl. Biol. 127:353-362.

2. Caten, C. E., and Jinks, J. L. 1968. Spontaneous variability of single isolates of Phytophthora infestans. I. Cultural variation. Can. J. Bot. 46: 329-348.

3. Chycoski, C. I., and Punja, Z. K. 1996. Characteristics of populations of Phytophthora infestans from potato in British Columbia and other regions of Canada during 1993 to 1995. Plant Dis. 80:579-589.

4. Colon, L. T., Turkensteen, L. J., Prummel, W., Budding, D. J., and Hoogendoorn, J. 1995. Durable resistance to late blight (Phytophthora infestans) in old potato cultivars. Eur. J. Plant Pathol. 101:387397.

5. Crosier, W. 1934. Studies in the biology of Phytophthora infestans (Mont.) De Bary. Cornell Univ. Exp. Stn. Mem. 155.

6. De Bary, A. 1873. The potato disease. J. Quekett Microsc. Club 3:139145 .

7. Drenth, A., Turkensteen, L. J., and Govers, F. 1993. The occurrence of the A2 mating type of Phytophthora infestans in the Netherlands: Significance and consequences. Neth. J. Plant Pathol. 99 (Suppl. 3): 57-67.
8. Duniway, J. M. 1983. Role of physical factors in the development of Phytophthora diseases. Pages 175-187 in: Phytophthora: Its Biology, Taxonomy, Ecology and Pathology. D. C. Erwin, S. Bartnicki-Garcia, and H. Tsao. The American Phytopathological Society, St. Paul, MN.

9. Fry, W. E., and Goodwin, S. B. 1997. Resurgence of the Irish potato famine fungus. Bioscience 47:363-371.

10. Fry, W. E., Goodwin, S. B., Dyer, A. T., Matuszak, J. M., Drenth, A., Tooley, P. W., Sujkowski, L. S., Koh, Y. J., Cohen, B. A., Spielman, L. J., Deahl, K. L., Inglis, D. A., and Sandlan, K. P. 1993. Historical and recent migrations of Phytophthora infestans: Chronology, pathways, and implications. Plant Dis. 77:653-661.

11. Glendinning, D., MacDonald, J. A., and Grainger, J. 1963. Factors affecting the germination of sporangia in Phytophthora infestans. Trans. Br. Mycol. Soc. 46:595-603.

12. Goodwin, S. B., Cohen, B. A., Deahl, K. L., and Fry, W. E. 1994. Migration from Northern Mexico as the probable cause of recent genetic changes in populations of Phytophthora infestans in the United States and Canada. Phytopathology 84:553-558.

13. Goodwin, S. B., Cohen, B. A., and Fry, W. E. 1994. Panglobal distribution of a single clonal lineage of the Irish potato famine fungus. Proc. Natl. Acad. Sci. USA 91:11591-11595.

14. Goodwin, S. B., Drenth, A., and Fry, W. E. 1992. Cloning and genetic analyses of two highly polymorphic, moderately repetitive nuclear DNAs from Phytophthora infestans. Curr. Genet. 22:107-115.

15. Goodwin, S. B., Schneider, R. E., and Fry, W. E. 1995. Use of celluloseacetate electrophoresis for rapid identification of allozyme genotypes of Phytophthora infestans. Plant Dis. 79:1181-1185.

16. Goodwin, S. B., Sujkowski, L. S., and Fry, W. E. 1995. Rapid evolution of pathogenicity within clonal lineages of the potato late blight disease fungus. Phytopathology 85:669-676.

17. Goodwin, S. B., Sujkowski, L. S., and Fry, W. E. 1996. Widespread distribution and probable origin of resistance to metalaxyl in clonal genotypes of Phytophthora infestans in the United States and Western Canada. Phytopathology 86:793-799.

18. Goth, R. W., and Keane, J. 1997. A detached-leaf method to evaluate late blight resistance in potato and tomato. Am. Potato J. 74:347-352.

19. Harrison, J. G. 1992. Effects of the aerial environment on late blight of potato foliage-A review. Plant Pathol. 41:384-416.

20. Harrison, J. G. 1995. Factors involved in the development of potato late blight disease (Phytophthora infestans). Pages 215-236 in: Potato Ecology and Modelling of Crops Under Conditions Limiting Growth. A. J. Haverkort and D. K. L. MacKerron, eds. Kluwer Academic Publishers, Dordrecht, Netherlands.

21. Harrison, J. G., and Lowe, R. 1989. Effects of humidity and air speed on sporulation of Phytophthora infestans on potato leaves. Plant Pathol. 38: 585-591.

22. Hartill, W. F. T., Young, K., Allan, D. J., and Henshall, W. R. 1990. Effects of temperature and leaf wetness on the potato late blight. N.Z. J. Crop Hortic. Sci. 18:181-184.

23. Hirst, J. M., and Stedman, O. J. 1960. The epidemiology of Phytophthora infestans. I. Climate, ecoclimate and the phenology of disease outbreak. Ann. Appl. Biol. 48:471-488.

24. Kadish, D., and Cohen, Y. 1988. Competition between metalaxyl-sensitive and metalaxyl-resistant isolates of Phytophthora infestans in the absence of metalaxyl. Plant Pathol. 37:558-564.

25. Kadish, D., and Cohen, Y. 1988. Fitness of Phytophthora infestans isolates from metalaxyl-sensitive and -resistant populations. Phytopathology 78: 912-915.

26. Kato, M., Mizubuti, E. S., Goodwin, S. B., and Fry, W. E. 1997. Sensitivity to protectant fungicides and pathogenic fitness of clonal lineages of Phytophthora infestans in the United States. Phytopathology 87:973-978.

27. Kuehl, R. O. 1994. Statistical Principles of Research Design and Analysis. Duxbury Press, Belmont, CA.

28. Lambert, D. H., and Currier, A. I. 1997. Differences in tuber rot development for North American clones of Phytophthora infestans. Am. Potato J. 74:39-43.

29. Legard, D. E., and Fry, W. E. 1996. Evaluation of field experiments by direct allozyme analysis of late blight lesions caused by Phytophthora infestans. Mycologia 88:608-612.

30. Legard, D. E., Lee, T. Y., and Fry, W. E. 1995. Pathogenic specialization in Phytophthora infestans: Aggressiveness on tomato. Phytopathology 85: 1356-1361.

31. Melhus, I. E. 1915. Germination and infection with the fungus of the late blight of potato (Phytophthora infestans). Agric. Exp. Stn. Univ. Wis. Res. Bull. 37:1-64.

32. Miller, J. S., Johnson, D. A., and Hamm, P. B. 1998. Aggressiveness of isolates of Phytophthora infestans from the Columbia Basin of Washington and Oregon. Phytopathology 88:190-197. 
33. Minogue, K. P., and Fry, W. E. 1981. Effect of temperature, relative humidity, and rehydration rate on germination of dried sporangia of Phytophthora infestans. Phytopathology 71:1181-1184.

34. Neter, J., Wasserman, W., and Kutner, M. H. 1990. Applied linear statistical models. Irwin, Boston.

35. Rotem, J., and Cohen, Y. 1974. Epidemiological patterns of Phytophthora infestans under semi-arid conditions. Phytopathology 64:711-714.

36. Sato, N. 1979. Effect of soil temperature on the field infection of potato tubers by Phytophthora infestans. Phytopathology 69:989-993.

37. Sato, N. 1994. Effect of sporulating temperature on the limit temperature in indirect germination of the sporangia of Phytophthora infestans. Ann. Phytopathol. Soc. Jpn. 60:60-65.

38. Sato, N. 1994. Effect of water temperature on direct germination of the sporangia of Phytophthora infestans. Ann. Phytopathol. Soc. Jpn. 60:162166.

39. Sato, N. 1994. Effect of some inorganic salts and hydrogen ion concen- tration on indirect germination of the sporangia of Phytophthora infestans. Ann. Phytopathol. Soc. Jpn. 60:441-447.

40. Snedecor, G. W., and Cochran, W. G. 1989. Statistical methods. Iowa State University Press, Ames.

41. Sujkowski, L. S., Goodwin, S. B., and Fry, W. E. 1996. Changes in specific virulence in Polish populations of Phytophthora infestans: 19851991. Eur. J. Plant Pathol. 102:555-561.

42. Van Oijen, M. 1992. Evaluation of breeding strategies for resistance and tolerance to late blight in potato by means of simulation. Neth. J. Plant Pathol. 98:3-11.

43. Warren, R. C., and Colhoun, J. 1975. Viability of sporangia of Phytophthora infestans in relation to drying. Trans. Br. Mycol. Soc. 64: 73-78.

44. Yamamoto, M., Aoki, S., and Suzuki, K. 1982. Induction of secondary zoosporangial formation in Phytophthora infestans. Trans. Mycol. Soc. Jpn. 23:211-215. 